\title{
Combining Ability and Heterosis Studies in Maize (Zea mays L.) under Kharif Season
}

\author{
Niranjan Kumar Chaurasia, R. B. P. Nirala* and Birender Singh \\ Department of Plant Breeding and Genetics, Bihar Agricultural University, Sabour, \\ Bhagalpur-813210, India \\ *Corresponding author
}

\section{Keywords}

General combining ability, Specific combining ability and standard heterosis

Article Info

Accepted:

20 October 2020 Available Online:

10 November 2020

\section{A B S T R A C T}

Present investigation was carried out in Kharif 2019 at Experimental Area, Department of Plant Breeding and Genetics, Bihar Agricultural University, Sabour, Bhagalpur with seven parental inbred lines and their twenty one $F_{1}$ 's generated through half diallel matting scheme during Rabi, 2018-19. The experiment was performed in randomized block design with three replications. Analysis of variance for diallel analysis (method II model I) revealed the mean squares due to genotypes, parents, hybrids and parent vs. hybrids were highly significant for all the fourteen quantitative characters under study. Analysis of variance for combining ability revealed the mean squares due to GCA and SCA were highly significant for all the characters study. The parents, P1 and P7 had significant to highly significant positive GCA effects for grain yield per plant and its attributes indicated these two parents were good general combiners for this trait. The cross combinations, $\mathrm{P} 1 \mathrm{x}$ P2, P1 x P4, P1 x P5, P2 x P3, P2 x P4, P2 x P5, P2 x P6, P3 x P4, P3 x P5, P3 x P6, P3 x $\mathrm{P} 7, \mathrm{P} 4 \times \mathrm{P} 5, \mathrm{P} 5 \times \mathrm{P} 6$, and $\mathrm{P} 6 \times \mathrm{P} 7$ had significant to highly significant SCA effects for grain yield per plant. The estimates of variance ratios of gca and sca were smaller than unity for all the characters study. The estimates of standard heterosis over the best check, DMRH-1308 for grain yield per plant revealed the top five cross combinations, namely P2 x P3, P6 x P7, P1 x P4, P3 x P4 and P3 x P6 exhibited highly significant positive standard heterosis. On the basis of standard heterosis, high SCA effects and high GCA effects for at least female parent for grain yield per plant and its attributes the hybrids, namely, P1 x P2, P1 x P4 and P1 x P5 were considered as promising experimental hybrids and may be exploited commercially after critical evaluation for their superior and stable performance over environments.

\section{Introduction}

Maize (Zea mays L., 2n=20) third major cereal crop that contribute to food security after rice and wheat. Besides the human food, it has great potential toward animal's feed and industrial raw material. Maize offers range of nutritional benefits and has clinical implications in various diseases (Murdia et al., 2016). According to National Collateral Management service, Special report (2017), nearly 13 per cent of the total maize 
consumed directly as food, 7 per cent as processed food, 47 per cent as poultry feed, 14 per cent as industrial raw materials, 13 per cent as animal feed and 6 per cent as export and others. In Bihar, maize is grown under the three crop growing seasons such as kharif, rabi and summer. Maize grown under kharif season faced allot of biotic and abiotic stresses such as irregular rain fall, water logging, pest and disease incidence etc which lead to severe reduction in grain yield. To boost the increment in grain yield heterosis breeding is one of an important tool. Selection of the parental lines used in hybridization programmes is critical task for breeder or researcher. Phenotypic selection of parental lines not always fulfils breeder's needs because phenotype is always associated with environments. Hence it is essential to select the parental lines on the basis of their genetic parameters. Combining ability analysis helps breeder to identify best combining inbred lines which may be used as parents for hybridization to exploit heterosis or to accumulate productive genes. Keeping this point the present investigation was carried out with the objectives, (I) to study the general combining ability and specific combining ability effects of parental inbred lines and hybrids, respectively and (II) to study the standard heterosis of hybrids.

\section{Materials and Methods}

The experimental material comprises seven inbred lines and their twenty one $F_{1}$ 's generated during Rabi 2018-19 using half diallel matting design and one hybrid as check. All these were evaluated for their agronomic performance during the Kharif, 2019 at experimental area, Bihar Agricultural College, Sabour. The experiment was carried out in randomized block design with three replications and $5 \mathrm{~m}$ row length having row to row distance $60 \mathrm{~cm}$ and plant to plant distance $20 \mathrm{~cm}$. The two seeds per hill were sown and after one week of germination thinning operation were performed to maintain single plant per hill. The recommended package of practices was followed for raising healthy crops. The data were recorded on fourteen quantitative traits on ten competitive plants from each replication viz., days to anthesis, days to silk, anthesis silking interval, days to 50 per cent physiological maturity, plant height $(\mathrm{cm})$, ear height $(\mathrm{cm})$, ear length $(\mathrm{cm})$, ear diameter $(\mathrm{cm})$, kernel row per ear, kernels per row, grains per plant, 1000-kernel weight $(\mathrm{g})$, shelling $(\%)$ and grain yield per plant. The mean values on different traits were analysed using INDOSTAT 9.2 software following the method II model I suggested by Griffing (1956).

\section{Results and Discussion}

Analysis of variance for diallel analysis (Method II Model 1) for fourteen quantitative traits (Table 1) revealed the mean squares due to genotypes, parents, hybrids and parent vs. hybrids were highly significant for all the characters studied indicated the existence of significant difference among the genotypes, parents, hybrids and parent vs hybrids. Analysis of variance for combining ability (Table 2) revealed the mean squares due to GCA and SCA were highly significant for all the characters studied indicated that all the traits might be governed by both additive and non-additive gene effects. The variance ratios of GCA and SCA (Table 5) were lower than unity indicated a non-additive gene effect was predominated for expression of characters studied.

\section{General combining ability (GCA) effects}

The estimates of GCA effects for different traits were either negative or positive (Table 3 ). For grain yield per plant positive GCA effects is desirable. The parents, P1 and P7 had highly significant positive GCA effects and were grouped as good general combiners for grain yield per plant. The parents, P2, P3 
and P6 were average general combiners, whereas, P4 and P5 were poor general combiners for grain yield per plant. The parent, P1 was also good general combiner for shelling per cent, grains per plant, kernels per row, kernel rows per ear, ear diameter and ear length and had highly significant positive GCA effects. The parent, P7 had highly significant positive GCA effects for the characters like shelling per cent, grains per plant, kernels per row and ear diameter and was grouped as good general combiner for these traits. Hence these two parents, P1 and P7 can be used directly for development of high yielding hybrids and synthetic by contributing desirable alleles. Significant positive GCA effects for inbred lines indicated that they are desirable parents for hybrid maize development and involvement in the maize breeding program as they can be source of good alleles in the process of varietal development (Rawi, 2016). Negative GCA effects are desirable for days to 50 per cent anthesis, days to 50 per cent silk, anthesis-silking interval and days to 50 per cent maturity. The parents, P1 had highly significant negative GCA effects for days to 50 per cent physiological maturity, anthesissilking interval, days to 50 per cent silk and days to 50 per cent anthesis, while, the parent, P6 had significant to highly significant negative GCA effects for anthesis-silking interval, days to 50 per cent silk and days to 50 per cent anthesis. Hence these two parents, P1 and P6 were good general combiners these traits and can be used as parent for development of early maturing hybrids. For the plant height, negative GCA effects are desirable. The parent, P3 had highly significant negative GCA effects for plant height and ear height and was grouped as good general combiner for these traits. These finding are close conformity with the results of Elmyhun et al., (2020), Singh et al., (2019), Kumar et al., (2017), Singh et al., (2017), Kumar and Babu (2016), Kumar et al., (2015), Dar et al., (2015), Gouda et al., (2013)

\section{Specific Combining Ability (SCA) effects}

The estimates of specific combining ability (SCA) effects for fourteen quantitative traits (Table 4) revealed that the cross combinations, namely, P2 x P3, P3 x P4, P3 x P6, P1 x P4, P6 x P7, P2 x P6, P3 x P5, P5 x $\mathrm{P} 6, \mathrm{P} 2 \times \mathrm{P} 4, \mathrm{P} 2 \times \mathrm{P} 5, \mathrm{P} 4 \times \mathrm{P} 5, \mathrm{P} 3 \times \mathrm{P} 7, \mathrm{P} 1 \times$ $\mathrm{P} 5$ and $\mathrm{P} 1 \mathrm{x} \mathrm{P} 2$ had significant to highly significant positive SCA effects for grain yield per plant and were grouped as good specific combinations for this traits. All these hybrids also exhibited significant to highly significant positive SCA effects for one or more yield contributing characters. High estimates of SCA effects for these cross combination revealed the preponderance of non-additive gene effects and may be exploited commercially for this trait after critical evaluation over locations/ years. Negative SCA effects are desirable for days to 50 per cent anthesis, days to 50 per cent silk, anthesis-silking interval and days to 50 per cent maturity for earliness. The top five cross combinations exhibited highly significant negative SCA effects were P2 x P6, P6 x P7, P1 x P4, P3 x P4 and P4 x P5 for days to 50 per cent anthesis and P6 x P7, P1 x P4, P3 x $\mathrm{P} 4, \mathrm{P} 2 \times \mathrm{P} 6$ and $\mathrm{P} 2 \times \mathrm{P} 7$ for days to 50 per cent silk. Almost all the cross combinations except P1 x P2, P1 x P7, P2 x P6, P3 x P55, P4 $x$ P5 and P5 x P7 exhibited highly significant negative SCA effects for anthesissilking interval. Two cross combinations $\mathrm{P} 1 \mathrm{x}$ P4 and PP1 x P6 had highly significant negative SCA effects for days to 50 per physiological maturity. P4 x P6 exhibited highly significant negative SCA effects for plant height, whereas, none of the crosses exhibited significant negative SCA effects for ear height. Hence, all these hybrids were grouped as good specific combinations for their respective traits in desirable direction. 
Table.1 Analysis of variance for diallel analysis (Method II and Model I) for fourteen quantitative characters in maize

\begin{tabular}{|c|c|c|c|c|c|c|c|c|}
\hline \multirow[t]{2}{*}{ Source of variation } & \multirow[b]{2}{*}{ D. F. } & \multicolumn{7}{|c|}{ Mean squares } \\
\hline & & DA & DS & ASI & DPM & PH & $\mathrm{EH}$ & EL \\
\hline Genotypes & 27 & $42.71 * *$ & $62.38 * *$ & $3.65 * *$ & $72.99 * *$ & $1669.92 * *$ & $635.81 * *$ & $11.72 * *$ \\
\hline Parents & 6 & $27.05 * *$ & $54.00 * *$ & $7.71 * *$ & $39.87 * *$ & $1876.43 * *$ & $1022.87 * *$ & $12.20 * *$ \\
\hline Hybrids & 20 & $13.20 * *$ & $20.76 * *$ & $1.90 * *$ & $59.42 * *$ & $407.07 * *$ & $213.59 * *$ & $2.72 * *$ \\
\hline Parent Vs. Hybrids & 1 & $726.92 * *$ & $945.02 * *$ & $14.29 * *$ & $543.25 * *$ & $25687.94 * *$ & $6757.83 * *$ & $188.95 * *$ \\
\hline Error & 54 & 1.54 & 1.54 & 0.00 & 11.51 & 67.74 & 38.43 & 0.81 \\
\hline
\end{tabular}

$* \& * *$ : level of significance at $5 \%$ and $1 \%$, respectively. DA: Days to 50 per cent anthesis, DS: days to 50 per cent silk, ASI: anthesis-silking interval, DPM: days to 50 per cent physiological maturity, PH: plant height, EH: ear height, EL: ear length

Contd. Table.1 Analysis of variance for diallel analysis (Method II and Model I) for fourteen quantitative characters in maize

\begin{tabular}{|c|c|c|c|c|c|c|c|c|}
\hline \multirow[t]{2}{*}{ Source of variation } & \multirow[t]{2}{*}{ D. F. } & \multicolumn{7}{|c|}{ Mean squares } \\
\hline & & ED & KRPE & KPR & GPP & $1000-\mathrm{KW}$ & SP & GYP \\
\hline Genotypes & 27 & $0.77 * *$ & $24.48 * *$ & $135.39 * *$ & $34258.41 * *$ & $1944.78 * *$ & $327.45 * *$ & $2691.46^{* *}$ \\
\hline Parents & 6 & $1.24 * *$ & $48.49 * *$ & $171.89 * *$ & $27995.96^{* *}$ & $2514.66^{* *}$ & $771.57 * *$ & $1641.24 * *$ \\
\hline Hybrids & 20 & $0.12 * *$ & $2.51 * *$ & $21.04 * *$ & $6664.28 * *$ & $1643.47 * *$ & $9.65 * *$ & $520.63 * *$ \\
\hline Parent Vs. Hybrids & 1 & $11.11 * *$ & $319.88 * *$ & $2203.55 * *$ & $623715.80 * *$ & $4551.75^{* *}$ & $4018.81 * *$ & $52409.49 * *$ \\
\hline Error & 54 & 0.01 & 0.317 & 5.17 & 870.09 & 415.00 & 3.17 & 106.83 \\
\hline
\end{tabular}

$* \& * *$ : level of significance at $5 \%$ and $1 \%$, respectively. ED: ear diameter, KRPE: kernel rows per ear, KPR: kernels per row, GPP: grains per plant, 1000-kw:

1000-kernel weight, SP: shelling per cent and GYP: grain yield per plant

Table.2 Analysis of variance for combining ability for fourteen quantitative characters in maize

\begin{tabular}{|c|c|c|c|c|c|c|c|c|c|c|c|c|c|c|c|}
\hline \multirow[t]{2}{*}{ Sources } & \multirow[b]{2}{*}{ D. F. } & \multicolumn{14}{|c|}{ Mean Squares } \\
\hline & & DA & DS & ASI & DPM & $\mathrm{PH}$ & $\mathrm{EH}$ & EL & ED & KRPE & KPR & GPP & $1000-K W$ & SP & GYP \\
\hline GCA & 6 & $16.68^{\star *}$ & $33.20^{* *}$ & $3.03^{* *}$ & $59.49^{\star *}$ & $687.27^{* *}$ & $431.03^{\star *}$ & $2.03^{* *}$ & $0.14^{* *}$ & $6.40^{* *}$ & $44.33^{\star *}$ & $8265.13^{\star *}$ & $1185.15^{\star *}$ & $146.08^{\star *}$ & $280.24^{\star *}$ \\
\hline SCA & 21 & $13.54^{\star *}$ & $17.25^{\star \star}$ & $0.70^{\star *}$ & $14.29^{\star \star}$ & $519.32^{\star *}$ & $149.34^{\star *}$ & $4.44^{\star *}$ & $0.29^{\star *}$ & $8.66^{\star *}$ & $45.36^{\star *}$ & $12320.71^{* *}$ & $494.86^{\star \star}$ & $98.60^{* \star}$ & $1073.41^{* *}$ \\
\hline Error & 54 & 0.52 & 0.52 & 0.00 & 3.84 & 22.58 & 12.81 & 0.27 & 0.01 & 0.11 & 1.72 & 290.03 & 138.33 & 1.06 & 35.61 \\
\hline
\end{tabular}


Table.3 Estimates of general combining ability (GCA) effects of seven parental inbred lines for fourteen quantitative characters in maize

\begin{tabular}{|c|c|c|c|c|c|c|c|}
\hline Sources & DA & DS & ASI & DPM & PH & EH & EL \\
\hline P1 & $-2.70 * *$ & $-3.76 * *$ & $-1.06 * *$ & $-5.70 * *$ & 2.56 & $5.11 * *$ & $0.66^{* *}$ \\
\hline P2 & $0.52 *$ & $0.79 * *$ & $0.27 * *$ & 0.82 & $4.14 * *$ & -1.14 & $-0.83 * *$ \\
\hline P3 & $1.23^{* *}$ & $1.94 * *$ & $0.71 * *$ & 1.19 & $-18.63 * *$ & $-14.26^{* *}$ & -0.05 \\
\hline P4 & $1.23^{* *}$ & $1.50 * *$ & $0.27 * *$ & 0.45 & $4.70 * *$ & $2.81 *$ & 0.31 \\
\hline P5 & -0.29 & $-0.69 * *$ & $-0.40 * *$ & 0.52 & 1.82 & -1.65 & -0.06 \\
\hline P6 & $-0.48 *$ & $-0.54 *$ & $-0.06 * *$ & 0.67 & $7.58 * *$ & $3.12 * *$ & -0.27 \\
\hline P7 & $0.49 *$ & $0.76 * *$ & $0.27 * *$ & $2.04 * *$ & -2.16 & $6.02 * *$ & 0.25 \\
\hline SE(gi) & 0.22 & 0.22 & 0.00 & 0.60 & 1.47 & 1.11 & 0.16 \\
\hline SE(gi-gj) & 0.34 & 0.34 & 0.00 & 0.92 & 2.24 & 1.69 & 0.25 \\
\hline
\end{tabular}

* \& **: level of significance at $5 \%$ and $1 \%$, respectively. DA: Days to 50 per cent anthesis, DS: days to 50 per cent silk, ASI: anthesis-silking interval, DPM: days to 50 per cent physiological maturity, PH: plant height, EH: ear height, EL: ear length.

Contd. Table.3 Estimates of general combining ability (GCA) effects of seven parental inbred lines for fourteen quantitative characters in maize

\begin{tabular}{|c|c|c|c|c|c|c|c|}
\hline Sources & ED & KRPE & KPR & GPP & 1000-KW & SP & GYP \\
\hline P1 & $0.07 * *$ & $1.19 * *$ & $2.46 * *$ & $37.65^{* *}$ & $-15.58 * *$ & $1.26^{* *}$ & $7.14 * *$ \\
\hline P2 & $0.167 * *$ & $0.72 * *$ & $-0.85^{*}$ & 2.31 & -0.96 & 0.02 & 0.64 \\
\hline P3 & $-0.22 * *$ & $-1.43 * *$ & $-2.63 * *$ & $-17.29 * *$ & -2.53 & $-7.59 * *$ & -2.94 \\
\hline P4 & 0.01 & -0.16 & -0.53 & $-29.67 * *$ & $10.76 * *$ & -0.02 & $-6.39 * *$ \\
\hline P5 & $-0.08 * *$ & $-0.33 * *$ & $-1.02 *$ & $-17.95 * *$ & 1.69 & $-1.96 * *$ & $-4.28 *$ \\
\hline P6 & 0.01 & $0.27 * *$ & $-1.07 *$ & $-21.25 * *$ & $17.45^{* *}$ & $3.95 * *$ & -2.12 \\
\hline P7 & $0.04 *$ & $-0.26^{*}$ & $3.65 * *$ & $46.20 * *$ & $-10.83^{* *}$ & $4.33 * *$ & $7.95 * *$ \\
\hline SE(gi) & 0.02 & 0.10 & 0.41 & 5.26 & 3.63 & 0.32 & 1.85 \\
\hline SE(gi-gj) & 0.03 & 0.15 & 0.62 & 8.03 & 5.55 & 0.48 & 2.81 \\
\hline
\end{tabular}

$* \& * *$ : level of significance at $5 \%$ and $1 \%$, respectively. ED: ear diameter, KRPE: kernel rows per ear, KPR: kernels per row, GPP: grains per plant,

1000-kw: 1000-kernel weight, SP: shelling per cent and GYP: grain yield per plant. 
Table.4 Estimate of specific combining ability (SCA) effects for fourteen quantitative characters in maize

\begin{tabular}{|c|c|c|c|c|c|c|c|}
\hline F1's & DA & DS & ASI & DPM & PH & EH & EL \\
\hline P1 x P2 & $-1.78^{*}$ & $-1.56^{\star}$ & $0.22^{\star *}$ & 0.43 & -1.01 & -5.85 & 0.27 \\
\hline P1 x P3 & -1.15 & $-1.37^{\star}$ & $-0.22^{\star *}$ & -1.28 & 0.88 & -0.67 & -0.27 \\
\hline P1 x P4 & $-3.48^{* *}$ & $-4.26^{\star *}$ & $-0.78^{* *}$ & $-5.87^{* *}$ & $15.32^{* *}$ & $9.14^{* *}$ & $0.98^{*}$ \\
\hline P1 x P5 & $-1.96^{\star *}$ & $-2.07^{\star *}$ & $-0.11^{* *}$ & -1.28 & $10.14^{*}$ & $8.01^{*}$ & 0.82 \\
\hline P1 x P6 & -0.78 & -1.22 & $-0.44^{* *}$ & $-5.76^{\star *}$ & 3.18 & -2.67 & -0.55 \\
\hline P1 x P7 & -0.41 & -0.19 & $0.22^{\star \star}$ & -2.13 & 6.31 & 2.88 & 0.25 \\
\hline P2 $x$ P3 & $-2.04^{\star *}$ & $-2.59^{\star *}$ & $-0.56^{\star \star}$ & 0.20 & $19.80^{\star *}$ & $15.76^{\star *}$ & $2.12^{\star *}$ \\
\hline P2 x P4 & $-2.04^{\star *}$ & $-2.19^{\star *}$ & $-0.11^{* *}$ & -2.06 & $11.39^{*}$ & $6.94^{*}$ & 0.36 \\
\hline P2 x P5 & $-2.19^{* \star}$ & $-2.63^{\star *}$ & $-0.44^{\star *}$ & -3.46 & $18.59^{\star \star}$ & $13.78^{* *}$ & $1.53^{\star *}$ \\
\hline P2 x P6 & $-3.67^{* *}$ & $-3.44^{\star *}$ & $0.22^{\star *}$ & -2.94 & $11.84^{*}$ & $6.73^{*}$ & $1.89^{\star *}$ \\
\hline P2 x P7 & $-1.63^{*}$ & $-2.74^{\star *}$ & $-1.11^{* *}$ & -2.65 & $10.51^{*}$ & $6.96^{*}$ & 0.52 \\
\hline P3 x P4 & $-3.07^{* *}$ & $-3.63^{\star *}$ & $-0.56^{\star *}$ & -2.426 & $24.82^{\star \star}$ & $13.58^{* *}$ & $2.48^{\star *}$ \\
\hline P3 x P5 & $-1.89^{* *}$ & -0.78 & $1.11^{\star *}$ & -1.83 & $19.71^{* *}$ & 5.40 & $1.69^{\star *}$ \\
\hline P3 x P6 & $-1.37^{*}$ & $-2.59^{\star *}$ & $-1.22^{\star \star}$ & 0.69 & $20.24^{\star *}$ & $6.70^{*}$ & $2.45^{\star *}$ \\
\hline P3 x P7 & 0.33 & -0.22 & $-0.56^{\star *}$ & 1.65 & 7.76 & 3.58 & 0.90 \\
\hline P4 x P5 & $-2.89^{\star \star}$ & $-2.33^{\star *}$ & $0.55^{\star \star}$ & -1.76 & 8.45 & 3.11 & 0.80 \\
\hline P4 x P6 & $1.96^{* *}$ & 1.19 & $-0.78^{* *}$ & -0.24 & $-14.10^{\star *}$ & -2.85 & $-1.65^{\star *}$ \\
\hline P4 x P7 & $-1.67^{*}$ & $-1.78^{*}$ & $-0.11^{* *}$ & 0.39 & 2.62 & 2.76 & 0.47 \\
\hline P5 x P6 & $-1.85^{\star *}$ & $-1.96^{\star *}$ & $-0.11^{* *}$ & -0.98 & $16.71^{\star *}$ & 1.96 & $1.09^{*}$ \\
\hline P5 x P7 & -0.48 & 0.07 & $0.56^{\star \star}$ & 1.65 & 0.17 & 1.04 & 0.39 \\
\hline P6 x P7 & $-3.63^{\star *}$ & $-4.41^{\star *}$ & $-0.78^{\star \star}$ & -1.17 & $18.70^{\star *}$ & $12.48^{* *}$ & $1.65^{\star *}$ \\
\hline SE(Sij) & 0.64 & 0.64 & 0.00 & 1.76 & 4.27 & 3.21 & 0.47 \\
\hline SE(Sij-Sik) & 0.96 & 0.96 & 0.00 & 2.61 & 6.34 & 4.77 & 0.69 \\
\hline SE(Sij-Skl) & 0.89 & 0.90 & 0.00 & 2.44 & 5.93 & 4.46 & 0.65 \\
\hline
\end{tabular}

* \& **: level of significance at $5 \%$ and $1 \%$, respectively. DA: Days to 50 per cent anthesis, DS: days to 50 per cent silk, ASI: anthesis-silking interval, DPM: days to 50 per cent physiological maturity, PH: plant height, EH: ear height, EL: ear length. 
Contd. Table.4 Estimate of specific combining ability (SCA) effects for fourteen quantitative characters in maize

\begin{tabular}{|c|c|c|c|c|c|c|c|}
\hline F1's & ED & KRPE & KPR & GPP & 1000-KW & SP & GYP \\
\hline P1 x P2 & 0.13 & -0.14 & 2.41 & $41.68^{*}$ & 8.47 & $2.34^{*}$ & $11.91^{*}$ \\
\hline P1 x P3 & $0.42^{\star *}$ & $2.42^{\star \star}$ & $2.85^{\star}$ & $39.34^{*}$ & 3.23 & $10.44^{\star *}$ & 4.76 \\
\hline P1 x P4 & 0.07 & 0.08 & $3.76^{\star *}$ & $78.13^{\star \star}$ & 8.35 & $2.40^{*}$ & $25.46^{* *}$ \\
\hline P1 x P5 & $0.15^{\star}$ & $1.72^{\star \star}$ & 2.18 & $81.94^{\star \star}$ & -18.52 & $3.04^{* \star}$ & $13.68^{*}$ \\
\hline P1 x P6 & 0.03 & -0.36 & -0.90 & -4.89 & 6.99 & -0.52 & -1.52 \\
\hline P1 x P7 & 0.06 & $-0.76^{\star}$ & 0.75 & 4.45 & 18.13 & -0.35 & 5.88 \\
\hline P2 x P3 & $0.75^{\star *}$ & $3.76^{\star *}$ & $5.94^{* *}$ & $89.55^{\star \star}$ & $34.55^{\star *}$ & 10.01 ** & $37.38^{* *}$ \\
\hline P2 x P4 & $0.19^{* *}$ & $0.88^{\star *}$ & 2.44 & $69.47^{\star \star}$ & -1.54 & $3.32^{* \star}$ & $19.18^{* *}$ \\
\hline P2 x P5 & $0.28^{* *}$ & $1.92^{\star *}$ & $5.31^{* *}$ & $85.14^{\star \star}$ & -14.00 & $5.42^{* *}$ & $17.65^{\text {** }}$ \\
\hline P2 x P6 & $0.18^{* *}$ & -0.02 & $4.05^{\star \star}$ & $72.51^{\star \star}$ & 1.24 & -0.06 & $23.55^{\text {** }}$ \\
\hline P2 x P7 & -0.02 & 0.25 & 1.24 & 18.79 & $-29.62^{*}$ & 0.66 & -4.49 \\
\hline P3 x P4 & $0.55^{* *}$ & $2.70^{\star *}$ & $6.75^{\star *}$ & $97.46^{\star *}$ & 21.29 & $10.77^{* *}$ & $34.36^{* *}$ \\
\hline P3 x P5 & $0.51^{* *}$ & $3.41^{* *}$ & $6.66^{\star *}$ & $94.56^{\star \star}$ & -9.37 & $10.51^{\text {** }}$ & $22.02^{* *}$ \\
\hline P3 x P6 & $0.53^{* *}$ & $2.00^{\star *}$ & $5.86^{\star *}$ & $68.24^{\star *}$ & $32.61^{* *}$ & $8.04^{* \star}$ & $27.77^{\star *}$ \\
\hline P3 x P7 & $0.30^{* *}$ & $2.53^{\star *}$ & $4.62^{* *}$ & $65.12^{\star \star}$ & 3.35 & $8.95^{\star \star}$ & $14.07^{*}$ \\
\hline P4 x P5 & $0.13^{*}$ & $1.20^{\star \star}$ & $3.06^{*}$ & $81.19^{\star \star}$ & -21.45 & $3.69^{* *}$ & $15.70^{* *}$ \\
\hline P4 x P6 & $-0.36^{\star *}$ & $-1.01^{* *}$ & $-3.12^{*}$ & $-52.91^{* *}$ & -16.81 & 1.32 & $-20.59^{* *}$ \\
\hline P4 x P7 & -0.06 & 0.46 & 2.07 & 21.11 & -0.80 & $2.69^{* \star}$ & 6.50 \\
\hline P5 x P6 & $0.35^{* *}$ & $1.04^{* *}$ & 1.92 & 29.03 & $31.92^{* *}$ & -0.25 & $20.61^{* *}$ \\
\hline P5 x P7 & 0.10 & $0.76^{*}$ & -0.46 & -17.95 & 19.27 & 1.15 & 4.58 \\
\hline P6 x P7 & 0.13 & $0.82^{*}$ & $4.73^{\star *}$ & $82.82^{\star \star}$ & 11.98 & 0.29 & $24.42^{* *}$ \\
\hline SE(Sij) & 0.06 & 0.29 & 1.18 & 15.29 & 10.56 & 0.92 & 5.36 \\
\hline SE(Sij-Sik) & 0.09 & 0.43 & 1.75 & 22.71 & 15.68 & 1.37 & 7.96 \\
\hline SE(Sij-Skm) & 0.09 & 0.41 & 1.64 & 21.24 & 14.70 & 1.28 & 7.44 \\
\hline
\end{tabular}

* \& **: level of significance at $5 \%$ and $1 \%$, respectively. ED: ear diameter, KRPE: kernel rows per ear, KPR: kernels per row, GPP: grains per plant, 1000-kw: 1000-kernel weight, SP: shelling per cent and GYP: grain yield per plant. 
Table.5 Estimates of components of variance for fourteen quantitative characters in maize

\begin{tabular}{|l|c|c|c|c|c|c|c|}
\hline \multirow{2}{*}{ Sources } & \multicolumn{9}{|c|}{ Mean squares } & \multicolumn{2}{c|}{} \\
\cline { 2 - 9 } & DA & DS & ASI & DPM & PH & EH \\
\hline $\boldsymbol{\sigma}^{\mathbf{2}}$ gca & 1.80 & 3.63 & 0.34 & 10.18 & 73.85 & 46.47 & 0.20 \\
\hline $\boldsymbol{\sigma}^{\mathbf{2}}$ sca & 13.02 & 16.74 & 0.70 & 0.45 & 496.74 & 136.53 & 4.17 \\
\hline $\boldsymbol{\sigma}^{\mathbf{2}} \mathbf{g c a} / \boldsymbol{\sigma}^{\mathbf{2}}$ sca & 0.14 & 0.22 & 0.48 & 0.15 & 0.34 & 0.05 \\
\hline
\end{tabular}

DA: Days to 50 per cent anthesis, DS: days to 50 per cent silk, ASI: anthesis-silking interval, DPM: days to 50 per cent physiological maturity, PH: plant height, EH: ear height, EL: ear length.

Contd. Table.5 Estimates of components of variance for fourteen quantitative characters in maize

\begin{tabular}{|c|c|c|c|c|c|c|c|}
\hline \multirow{2}{*}{ Sources } & \multicolumn{7}{|c|}{ Mean squares } \\
\hline & ED & KRPE & KPR & GPP & $1000-\mathrm{KW}$ & SP & GYP \\
\hline$\sigma^{2}$ gca & 0.01 & 0.70 & 4.73 & 886.12 & 116.31 & 16.11 & 27.18 \\
\hline$\sigma^{2}$ sca & 0.29 & 8.56 & 43.64 & 12030.68 & 356.53 & 97.54 & 1037.81 \\
\hline$\sigma^{2}$ gca $/ \sigma^{2}$ sca & 0.05 & 0.08 & 0.11 & 0.07 & 0.33 & 0.17 & 0.03 \\
\hline
\end{tabular}

ED: ear diameter, KRPE: kernel rows per ear, KPR: kernels per row, GPP: grains per plant, 1000-KW: 1000-kernel weight, SP: shelling per cent and GYP: grain yield per plant 
Table.6 Estimates of standard heterosis over the best check, DMRH-1308 for grain yield and its attributes

\begin{tabular}{|c|c|c|c|c|c|c|c|c|c|c|c|c|c|c|c|}
\hline $\begin{array}{c}\text { Sl. } \\
\text { No } \\
\text {. }\end{array}$ & $F_{1}$ 's & DA & DS & ASI & DPM & PH & EH & EL & ED & KRPE & KPR & GPP & $\begin{array}{c}\text { 1000- } \\
\text { KW }\end{array}$ & SP & GYP \\
\hline 1 & $\mathrm{P} 1 \times \mathrm{P} 2$ & -3.57 & $-9.50 * *$ & $-100.00 * *$ & -1.44 & $11.88 * *$ & 9.33 & 2.72 & 0.91 & -3.23 & 11.43 & $25.87 * *$ & $23.52 * *$ & 1.37 & $52.43 * *$ \\
\hline 2 & $\mathrm{P} 1 \times \mathrm{P} 3$ & -1.19 & $-7.26 * *$ & $-100.00 * *$ & -2.88 & -0.77 & -1.77 & 4.6 & -1.49 & -0.46 & 5.79 & 17.88 & $20.23^{*}$ & 1.99 & $36.10 * *$ \\
\hline 3 & $\mathrm{P} 1 \times \mathrm{P} 4$ & $-5.36 * *$ & $-12.85^{* *}$ & $-127.27 * *$ & $-8.63 * *$ & $22.09 * *$ & $35.79 * *$ & $16.97 * *$ & -4.38 & $-7.83^{*}$ & $18.49^{*}$ & $27.49 * *$ & $29.14 * *$ & 1.40 & $62.35^{* *}$ \\
\hline 4 & P1 x P5 & $-5.36 * *$ & $-12.85 * *$ & $-127.27 * *$ & -3.6 & $17.22 * *$ & $27.98 * *$ & $12.88 *$ & -4.88 & 2.3 & 9.74 & $33.15^{* *}$ & 11.75 & -0.21 & $47.63 * *$ \\
\hline 5 & P1 x P6 & -3.57 & $-11.17 * *$ & $-127.27 * *$ & $-8.27 * *$ & $16.49 * *$ & $19.72 * *$ & 0.67 & $-5.37^{*}$ & $-7.83^{*}$ & -3.53 & 0.34 & $31.72 * *$ & 2.70 & $27.79 *$ \\
\hline 6 & P1 x P7 & -1.19 & $-7.26^{* *}$ & $-100.00 * *$ & -2.88 & $12.49 * *$ & $31.54 * *$ & 10.87 & -3.97 & $-14.29 * *$ & $23.50 * *$ & $28.29 * *$ & $23.43 * *$ & 3.39 & $54.37 * *$ \\
\hline 7 & $\mathrm{P} 2 \times \mathrm{P} 3$ & 2.98 & -1.68 & $-72.73 * *$ & 5.76 & $11.65^{* *}$ & 12.45 & 11.57 & $9.01 * *$ & 5.53 & 4.8 & $23.30^{*}$ & $42.47 * *$ & -0.09 & $75.83 * *$ \\
\hline 8 & $\mathrm{P} 2 \times \mathrm{P} 4$ & 2.98 & -1.68 & $-72.73 * *$ & 2.52 & $20.68 * *$ & $23.98 * *$ & 0.75 & 0.91 & -5.53 & -1.13 & 11.48 & $31.43 * *$ & 1.00 & $42.89 * *$ \\
\hline 9 & $\mathrm{P} 2 \times \mathrm{P} 5$ & 0.00 & $-6.15^{* *}$ & $-100.00 * *$ & 1.08 & $23.30 * *$ & $27.31 * *$ & 6.92 & 0.83 & 0.46 & 8.96 & $21.45^{*}$ & $21.01 *$ & 1.20 & $43.78 * *$ \\
\hline 10 & P2 x P6 & -2.98 & $-7.26^{* *}$ & $-72.73 * *$ & 1.8 & $22.70 * *$ & $24.12 * *$ & 8.02 & 0.83 & $-8.76 * *$ & 3.39 & 15.65 & $36.01 * *$ & 1.73 & $56.04 * *$ \\
\hline 11 & $\mathrm{P} 2 \times \mathrm{P} 7$ & 2.38 & $-3.91 *$ & $-100.00 * *$ & 3.6 & $16.00 * *$ & $28.49 * *$ & 1.54 & -3.47 & $-10.60 * *$ & 11.5 & $20.65 *$ & 7.39 & 3.10 & $28.70 *$ \\
\hline 12 & $\mathrm{P} 3 \times \mathrm{P} 4$ & 2.38 & -2.23 & $-72.73 * *$ & 2.52 & $15.02 * *$ & $14.92 *$ & $23.06^{* * *}$ & 0.17 & $-7.83 *$ & 9.6 & 14.54 & $41.72 * *$ & 0.80 & $60.55^{* *}$ \\
\hline 13 & P3 x P5 & 1.79 & -1.12 & $-45.45^{* *}$ & 3.24 & $10.19 *$ & -2.74 & $14.14^{*}$ & -3.06 & -4.15 & 7.13 & 17.74 & $22.49 * *$ & -1.92 & $44.99 * *$ \\
\hline 14 & P3 x P6 & 2.38 & $-3.91 *$ & $-100.00 * *$ & 6.12 & $13.99 * *$ & 5.75 & $18.43 * *$ & -0.33 & $-9.68 * *$ & 3.53 & 6.96 & $50.44 * *$ & 2.34 & $57.02 * *$ \\
\hline 15 & P3 x P7 & $7.14 * *$ & 2.23 & $-72.73 * *$ & $8.63 * *$ & 0.54 & 5.43 & 10.46 & $-5.29 *$ & $-9.68 * *$ & $18.28^{*}$ & $30.38^{* *}$ & $22.59 * *$ & $3.94^{*}$ & $51.50 * *$ \\
\hline 16 & P4 x P5 & 0.00 & $-4.47 *$ & $-72.73 * *$ & 2.52 & $17.49 * *$ & $17.92 *$ & 10.05 & $-6.69 * *$ & $-10.60 * *$ & 0.78 & 8.37 & $23.07 * *$ & -0.99 & $30.12 *$ \\
\hline 17 & P4 x P6 & $8.33 * *$ & 1.68 & $-100.00 * *$ & 4.32 & 7.33 & $16.25^{*}$ & -10.44 & $-16.53 * *$ & $-21.66 * *$ & $-25.62 * *$ & $-41.64 * *$ & $32.95 * *$ & 3.40 & -21.82 \\
\hline 18 & P4 x P7 & 3.57 & -1.12 & $-72.73 * *$ & $6.47 *$ & $11.56^{* *}$ & $28.14 * *$ & 9.9 & $-8.43 * *$ & $-15.21 * *$ & 16.37 & 9.85 & $27.01 * *$ & $5.57 * *$ & $34.73 * *$ \\
\hline 19 & P5 x P6 & -1.19 & $-7.26 * *$ & $-100.00 * *$ & 3.6 & $24.24 * *$ & $16.74 *$ & 7.79 & -1.24 & $-8.76 * *$ & -6.35 & -7.55 & $52.15^{* *}$ & -0.96 & $44.08 * *$ \\
\hline 20 & P5 x P7 & 2.98 & -1.68 & $-72.73 * *$ & $7.91 *$ & 8.33 & $19.51 * *$ & 6.45 & $-6.78 * *$ & $-14.29 * *$ & 3.6 & -0.1 & $32.33 * *$ & 1.25 & $35.01 * *$ \\
\hline 21 & P6 x P7 & -2.98 & $-8.94 * *$ & $-100.00 * *$ & 5.04 & $23.03 * *$ & $42.17 * *$ & $14.55^{*}$ & -3.64 & $-9.68 * *$ & $25.34 * *$ & $35.38 * *$ & $36.43 * *$ & $7.51 * *$ & $68.49 * *$ \\
\hline
\end{tabular}

* \& **: level of significance at $5 \%$ and $1 \%$, respectively. DA: Days to 50 per cent anthesis, DS: days to 50 per cent silk, ASI: anthesis-silking interval, DPM: days to 50 per cent physiological maturity, PH: plant height, EH: ear height, EL: ear length, ED: ear diameter, KRPE: kernel rows per ear, KPR: kernels per row, GPP: grains per plant, 1000-KW: 1000-kernel weight, SP: shelling per cent and GYP: grain yield per plant. 
The promising cross combinations having significant to highly significant SCA effects in desirable direction could be exploited commercially after verifying their performance across environments. These results are in close conformity with the findings of Singh et al., (2019), Ambikabathy et al., (2019), Abhishek (2018), Dar et al., (2018), Singh et al., (2017), Kumar and Babu (2016), Kumar et al., (2015) and Gouda et al., (2013).

\section{Standard heterosis}

The estimates of standard heterosis over the check, DMRH-1308 for fourteen quantitative traits (Table 6) revealed the per cent of standard heterosis for grain yield ranged from -21.82 per cent $(\mathrm{P} 4 \times \mathrm{P} 6)$ to 75.83 per cent (P2 x P3). The top five cross combinations, $\mathrm{P} 2 \times \mathrm{P} 3, \mathrm{P} 6 \times \mathrm{P} 7, \mathrm{P} 1 \times \mathrm{P} 4, \mathrm{P} 3 \times \mathrm{P} 4$ and $\mathrm{P} 3 \times$ P6 had highest positive significant standard heterosis for grain yield per plant. These hybrids also exhibited significant to highly significant positive standard heterosis for one or more grain yield contributing traits. Hence, these hybrids can be exploited commercially after critical evaluation for their superiority and stability over the locations or years. Days to 50 per cent anthesis and days to 50 percent silk regulate the early flowering. The cross combinations, P1 x P4 and P1 x P5 had highly significant negative standard heterosis for days to 50 per cent anthesis. For days to 50 per cent silk, the cross combinations, namely, P1 x P2, P1 x P3, P1 x P4, P1 x P5, P1 x P6, P1 x P7, P2 x P5, P2 x P6, P2 x P7, P3 x P6, P4 x P5, P5 x P6 and P6 x P7 exhibited significant to highly negative standard heterosis, while, all the cross combinations had highly significant negative standard heterosis for anthesis-silking interval. Two cross combinations, P1 x P4 and P1 x P6 had highly significant negative standard heterosis for days to 50 per cent maturity. Hence these hybrids can be exploited earliness after critical testing over environments. Similar results were reported by Ambikabathy et al., (2019), Kumar and Babu (2016), Kumar et al., (2015), Ofori et al., (2015), Lahane et al., (2014).

Hence concluded, in the present study for grain yield per, the cross combinations, $\mathrm{P} 1 \mathrm{x}$ P2, P1 x P4 and P1 x P5 were identified as the best experimental hybrids on the basis of high standard heterosis, high SCA and high GCA for at least female parent. Hence, these hybrids may be exploited commercially after critical evaluation for their performance and stability over environments for this trait.

\section{Acknowledgement}

Authors thankful to Dean PGS, Bihar Agricultural University, Sabour, Bhagalpur for their financial support for accomplishing research works. Authors also thankful to publication committee to provide BAU communication number $896 / 201104$ for the manuscript.

\section{References}

Abhishek A (2018) Combining Ability and heterosis in maize (Zea mays L.) for yield and its associate traits, M.Sc. (Ag.) thesis, Bihar Agricultural University, Sabour, Bhagalpur.

Ambikabathy A, Jegadeesh S N, Thirusendura $\mathrm{S}$ D, Dhasarathan $\mathrm{M}$, Vairam $\mathrm{N}$, Renganathan G V, Mathivathana K M, Paranthaman M, Karthikeyan A, Vanniarajan C, Karthikeyan $\mathrm{G}$ and Senthil N (2019) Determination of Combining Ability and Heterosis for Yield and Yield Related Traits in Maize Hybrids Based on Line $\times$ Tester Analysis, Research Journal of Agricultural Sciences, 10(1): 215-220.

Dar Z A, Lone A A, Alie B A, Ahangar M A, Ali G, Abidi I, Gazal A and Lone R A 
(2018) Combining ability analysis for yield and yield contributing traits in Popcorn (Zea mays everta L.) under temperate conditions, Journal of Pharmacognosy and Phytochemistry, 7(1): 361-366.

Elmyhun M, Liyew C, Shita A and Andualem M (2020) Combining ability performance and heterotic grouping of maize (Zea mays) inbred lines in testcross formation in Western Amhara, North West Ethiopia, Cogent Food \& Agriculture, 6:1, 1727625.

Gouda R Kambe, Kage U K, Lohithaswa H C, Shekara B G, Shobha D (2013) Combining ability in maize (Zea mays L.), Molecular Plant Breeding, 3(4):116-127.

Griffing B (1956) Concept of general and specific combining ability in relation to diallel crossing system, Australian Journal of Biological Science, 9:463493.

Kumar A, Dadheech A, Kiran N, Bisen P and Kumar S (2017) Diallel Analysis of Combining Ability for Yield and Yield Contributing Traits over the Environments in Maize (Zea mays L.), International Journal of Current Microbiology and Applied Sciences, 6 (10): 196-208.

Kumar R, Mandal S S, Mishra A K, Smriti, Singh R and Kumar P (2015) Heterosis and combining ability for yield and its contributing traits of kharif maize (zea mays 1.), The Bioscan, 10(4): 20492056.

Kumar S V P and Babu D R (2016) Combining ability and heterosis in maize (Zea mays L.) for grain yield and yield components, International Journal of Agriculture, Environment and Biotechnology, 9(5): 763-772.

Lahane G, Chauhan R and Patel J M (2014) Combining ability and heterosis for yield and quality traits in quality protein maize. Journal of Agricultural research, 1(3): 135-142.

National Collateral Management service, Special report (2017)

Ofori A P, Ofori K, Obeng-Antwi K, Tengan K M L and Badu-Apraku B (2015) Combining ability and heterosis estimate of extra-early quality protein maize (QPM) single cross hybrids. Journal of Plant Breeding and Crop Science, 7(4): 87-93.

Rawi (2016) Relative performance and combining ability for yield and yield components in maize by using full diallel cross, International Journal of Current Research, 8(9): 37721-37728.

Singh B, Abhishek A, Nirala R B P, Mandal S $S$ and Ranjan $T$ (2019) Study of Combining Ability and Nature of Gene Action for Yield and Yield Related Traits in Maize (Zea mays L.), 33(2): 18.

Singh M, Dubey R B, Ameta K D, Haritwal S and Bhagchand (2017) Combining ability analysis for yield contributing and quality traits in yellow seeded late maturing maize (Zea mays L.) hybrids using Line $\mathrm{x}$ Tester, Journal of Pharmacognosy and Phytochemistry, 6(5): 112-118.

\section{How to cite this article:}

Niranjan Kumar Chaurasia, R. B. P. Nirala and Birender Singh. 2020. Combining Ability and Heterosis Studies in Maize (Zea mays L.) under Kharif Season. Int.J.Curr.Microbiol.App.Sci. 9(11): 2576-2586. doi: https://doi.org/10.20546/ijcmas.2020.911.312 